J. Lake Sci.(湖泊科学), 2017, 29(3): 617-624

DOI 10. 18307/2017. 0310

(c) 2017 by Journal of Lake Sciences

\title{
群体微囊藻附生细菌特性”
}

范琦, 肖惠杰, 吴强, 汪水娟, 李朋富**

(南京大学生命科学学院, 南京 210093)

摘 要: 附生细菌在微囊藻水华暴发过程中可能发挥了重要作用, 但是, 缺少关于微囊藻附生细菌特性的研究报道. 本文 从 4 种群体微囊藻( 铜绿微囊藻、惠氏微囊藻、水华微囊藻和坚实微囊藻) 中分离得到了 18 种附生细菌, 分别隶属于六大 类群: $\alpha$-变形菌纲、 $\beta$-变形菌纲、 $\gamma$-变形菌纲、放线菌纲、纤维粘网菌纲和黄杆菌纲.BIOLOG 板分析显示除了 $\alpha$-变形菌纲中 的 2 种细菌以及放线菌纲中的 1 种细菌以外, 其余细菌都能够利用 10 种以上的碳源. 18 种附生细菌中有 12 种具有趋化 性, 12 种具有疏水性, 14 种具有自聚能力. 除了黄杆菌纲菌株都具有亲水性外, 其余 5 大类群菌株都含有疏水性种类. 六大 类群中都含有具有趋化性和自聚能力的种类. 每种微囊藻中的大多数附生细菌种类都能利用 10 种以上的碳源, 都具有疏 水性. 每种微囊藻中至少有一组细菌表现出共聚能力. 除了水华微囊藻外, 其余 3 种微囊藻中附生细菌的大多数种类都具 有趋化性. 除了惠氏微囊藻外, 其余 3 种微囊藻的附生细菌中大多数种类都具有自聚能力. 结果显示细菌旺盛的代谢潜 力、趋化性、疏水性、自聚能力和共聚能力可能有利于附生细菌定殖于微囊藻群体.

关键词: 微囊藻; 附生细菌;多样性; 特性

\section{Characterization of epiphytic bacteria associated with colonial Microcystis}

\author{
FAN Qi, XIAO Huijie, WU Qiang, WANG Shuijuan \& LI Pengfu** \\ (School of Life Sciences, Nanjing University, Nanjing 210093, P.R.China)
}

\begin{abstract}
Epiphytic bacteria associated with colonial Microcystis may play an important role in the development of cyanobacterial bloom. However, information on characterization of Microcystis-associated bacteria is limited. In this study, eighteen Microcystis-associated bacteria which belonged to six bacterial groups: Alpha proteobacterium, Beta proteobacterium, Gamma proteobacterium, Actinobacteria, Cytophagia and Flavobacteriia, were isolated from four Microcystis species (M. wesenbergii, M. flos-aquae, M. firma and M. aeruginosa). Analysis by BIOLOG plate indicated that except for two isolates of Alpha proteobacterium and one isolate of Actinobacteria, the other isolates were capable of utilizing more than 10 carbon sources. Among 18 bacterial isolates, 12 isolates showed chemotaxis, 12 isolates displayed hydrophobicity, and 14 isolates exhibited autoaggregation ability. Except for Flavobacteri$i a$, other five groups contained hydrophobic bacterial isolates. All six groups contained bacterial isolates with chemotaxis and autoaggregation ability. Most of bacterial isolates associated with each Microcystis species were capable of utilizing more than 10 carbon sources, and exhibited hydrophobicity. For each Microcystis species, there was at least one group of bacterial isolates exhibiting coaggregation ability. Except for M. flos-aquae, most of bacterial isolates associated with each of other three Microcystis species showed chemotaxis. The results indicated that active metabolic potential, chemotaxis, hydrophobicity, and the abilities of autoaggregation and coaggregation might help the bacteria colonize the Microcystis colonies.
\end{abstract}

Keywords: Microcystis; epiphytic bacteria; diversity; characteristic

蓝藻水华是危害公共健康和环境安全的全球性问题.微囊藻 (Microcystis) 是在湖泊和水库中形成蓝藻水 华的最常见藻类 ${ }^{[1]}$. 在自然界中微囊藻多以群体形态存在,群体的形成有利于微囊藻垂直迁移和抵御浮游动 物的摄食, 群体微囊藻比单细胞微囊藻具有更高的生存竞争优势, 群体的形成是微囊藻形成优势并暴发水

* 国家自然科学基金项目 (31270447) 和国家重点基础研究发展计划“973” 项目 (2008CB418004) 联合资助. 2016 07-06 收稿; 2016-09-07 收修改稿. 范琦 (1993 ), 女, 硕士研究生;E-mail: fanqi1993@ yahoo.com.

** 通信作者;E-mail: pengfuli@ nju.edu.cn. 
华的关键机制之一 ${ }^{[1-2]}$.

附生细菌附着在微囊藻群体中生长, 包括变形菌 (Proteobacteria) 、拟杆菌 (Bacteroidetes) 和放线菌 (Actinobacteria $)$ 等, 其菌群组成不同于周围水体中的游离细菌菌群 ${ }^{[3-5]}$. 附生细菌可能促进或者抑制微囊藻生长, 诱导微囊藻群体的形成, 一些附生细菌能降解微囊藻毒素, 因此附生细菌在微囊藻水华暴发过程中可能发 挥了重要作用 ${ }^{[6-8]}$. 但是, 很少有关于微囊藻附生细菌特性的研究报道, 这阻碍了对附生细菌生态学功能的深 人理解. 为了探讨附生细菌定殖于微囊藻群体的机制, 本文从微囊藻群体中分离附生细菌, 并分析其组成、生 化特性、趋化性、细胞表面疏水性以及自聚 (autoaggregation) 和共聚 (coaggregation) 能力.

\section{1 材料与方法}

\section{1 微囊藻的培养与附生细菌的分离}

惠氏微囊藻 NJ-24( M. wesenbergii) 、坚实微囊藻 NJ-54 (M. firma)、铜绿微囊藻 NJ-177 ( M. aeruginosa) 和 水华微囊藻 $\mathrm{NJ}-159$ (M. flos-aquae) 于 2011 年 8 月从太湖采集和分离. 在 $50 \mathrm{ml}$ 三角瓶中加人无菌 BG-11 培 养基进行培养 ${ }^{[9]}, 4$ 株微囊藻在实验室培养过程中一直呈现群体形态, 培养温度为 $25^{\circ} \mathrm{C}$, 光照强度为 2500 lux, 光暗周期为 $12 \mathrm{~h}: 12 \mathrm{~h}$.

实验室中培养一年后, 在超净台中将微囊藻群体放在无菌的尼龙篎 (孔径 $20 \mu \mathrm{m}$ ) 上, 用无菌 BG-11 培 养基冲洗 10 次, 然后将群体微囊藻涂布在 $\mathrm{R}_{2} \mathrm{~A}$ 固体培养基上 ${ }^{[10]}, 30^{\circ} \mathrm{C}$ 恒温培养, 根据固体培养基上的菌落 数量计算各菌株在总菌落数中所占比例. 选取不同形态的菌落通过在 $\mathrm{R}_{2} \mathrm{~A}$ 平板上划线纯化.

\section{2 附生细菌的分子鉴定}

参考 Tillett 等的方法提取细菌 DNA ${ }^{[11]}$. 采用引物 $27 \mathrm{~F}\left(5^{\prime}\right.$-AGAGTTTGATCCTGGCTCAG $\left.-3^{\prime}\right)$ 和 $1492 \mathrm{R}$ (5'-GGTTACCTTGTTACGACTT- $\left.3^{\prime}\right)$ 扩增附生细菌的 $16 \mathrm{~S}$ rRNA 基因部分序列.PCR 扩增条件如下: $94^{\circ} \mathrm{C}$ 预变 性 $1 \mathrm{~min}$, 接着为 20 个循环反应 $\left(94^{\circ} \mathrm{C} 30 \mathrm{~s}, 50^{\circ} \mathrm{C} 30 \mathrm{~s}, 72^{\circ} \mathrm{C} 2 \mathrm{~min}\right)$, 最后在 $72^{\circ} \mathrm{C}$ 下延伸 $7 \mathrm{~min}$. PCR 产物由南 京金斯瑞生物科技有限公司完成测序, 所得 DNA 序列提交到 GenBank 中, 并用 BLAST 找到最相似的序列.

\section{3 附生细菌的生化特性}

收集对数期的细菌,用 $0.85 \% \mathrm{NaCl}$ 溶液洗涤 3 次, 并重悬于 $0.85 \% \mathrm{NaCl}$ 溶液中至 $\mathrm{OD}_{600} \approx 0.1$, 取 $150 \mu \mathrm{l}$ 的细菌悬液, 接种到 BIOLOG 生态板 (EcoPlates 1506, 美国 BIOLOG 公司) 中, 用 $150 \mu \mathrm{l}$ 的 $0.85 \% \mathrm{NaCl}$ 溶液 作对照, $30^{\circ} \mathrm{C}$ 下恒温培养. BIOLOG 板的每个孔中含有不同的碳源, 当细菌能利用碳源时 BIOLOG 板的孔中 呈现紫色, 否则, 就保持无色 ${ }^{[12]}$, 每个菌株进行 3 次以上重复验证.

\section{4 附生细菌的趋化性}

采用平板法检测细菌对于葡萄糖和氨基酸的趋化性 ${ }^{[13-14]}$. 固体培养基包含 $1 \times 10^{-2} \mathrm{~mol} / \mathrm{L}$ 的 $\mathrm{K}_{2} \mathrm{HPO}_{4} 、 1 \times$ $10^{-3} \mathrm{~mol} / \mathrm{L}$ 的 $\mathrm{MgSO}_{4} 、 1 \times 10^{-3} \mathrm{~mol} / \mathrm{L}$ 的 $(\mathrm{NH} 4)_{2} \mathrm{SO}_{4} 、 1 \times 10^{-4} \mathrm{~mol} / \mathrm{L}$ 的 EDTA $3 \times 10^{-5} \mathrm{~mol} / \mathrm{L}$ 的蛋氨酸、 $1 \times 10^{-5}$ $\mathrm{mol} / \mathrm{L}$ 的 D-葡萄糖和 $0.2 \%$ 琼脂, $\mathrm{pH}$ 值调至 7.0. 收集培养到对数期的细菌, 用 $0.85 \% \mathrm{NaCl}$ 溶液连续洗涤 3 次, 重悬细菌.将吸取 $10 \mu \mathrm{l}$ 重悬菌液的枪头垂直插人固体培养基, 菌液慢慢渗人琼脂培养基中. $30^{\circ} \mathrm{C}$ 下恒温 培养, 细菌从接种点为中心向外圆形扩散生长, 以 4 天后检测的圆形直径大小来反映细菌的趋化性强弱, 每 种细菌重复 3 次实验.

\section{5 附生细菌的细胞表面疏水性}

采用 MATH 法来测定细胞疏水性 ${ }^{[15]}$, 收集培养 $48 \mathrm{~h}$ 的细菌, 用无菌磷酸盐缓冲液 ( PBS, $0.01 \mathrm{~mol} / \mathrm{L}, \mathrm{pH}$ 7.0 ) 洗涤 3 次并重悬于 $\mathrm{PBS}$ 中至 $\mathrm{OD}_{600} \approx 1.0$, 取 $4 \mathrm{ml}$ 洗涤后的细菌悬浮液加 $4 \mathrm{ml}$ 正十六烷于试管中涡旋混 合 $1 \mathrm{~min}$, 静置 $10 \mathrm{~min}$, 测定下层水相 $\mathrm{OD}_{600}$ 值.

$$
\text { 细菌细胞表面疏水性 }(\%)=\frac{\left(\mathrm{OD}_{\mathrm{i}}-\mathrm{OD}_{\mathrm{f}}\right)}{\mathrm{OD}_{\mathrm{i}}} \times 100 \%
$$

式中, $\mathrm{OD}_{\mathrm{i}}$ 是初始细菌悬液的 $\mathrm{OD}_{600}$ 值, $\mathrm{OD}_{\mathrm{f}}$ 是与十六烷混合后下层水相的 $\mathrm{OD}_{600}$ 值, 每种细菌做 3 次重复实验.

\section{6 附生细菌的自聚和共聚能力}

采用分光光度法分析细菌的自聚和共聚能力 ${ }^{[16]}$. 收集培养 $48 \mathrm{~h}$ 的细菌, PBS 洗涤 3 次并重悬细菌至 $\mathrm{OD}_{600} \approx 1.0$, 每种菌液取 $4 \mathrm{ml}$ 加人到比色血中, 室温下静置 $0 、 1 、 4$ 和 $24 \mathrm{~h}$ 后检测细菌悬浮液的 $\mathrm{OD}_{600}$ 值. 


$$
\text { 自聚能力 }(\%)=\frac{\left(A_{0}-A_{\mathrm{t}}\right)}{A_{\mathrm{t}}} \times 100 \%
$$

式中, $A_{0}$ 是细菌悬浮液的初始 $\mathrm{OD}_{600}$ 值, $A_{\mathrm{t}}$ 是静置后细菌悬浮液的 $\mathrm{OD}_{600}$ 值.

共聚能力分析时细菌悬液的制备同上, 同种微囊藻中分离得到的附生细菌每两株一组, 各取 $2 \mathrm{ml}$ 细菌 悬浮液加人到同一比色血中混匀, 同时每种细菌各取 $4 \mathrm{ml}$ 分别加人到一个比色血中作为对照, 室温静置 1 、 $4 、 24 \mathrm{~h}$ 后, 检测混合菌液和单个菌液的 $\mathrm{OD}_{600}$ 值.

$$
\text { 共聚能力 }(\%)=\frac{\left[\left(A_{\mathrm{x}}+A_{\mathrm{y}}\right)-2 \times A_{\mathrm{xy}}\right]}{\left(A_{\mathrm{x}}+A_{\mathrm{y}}\right)} \times 100 \%
$$

式中, $A_{x}$ 和 $A_{y}$ 分别是两种细菌在对照比色血中的 $\mathrm{OD}_{600}$ 值, $A_{x y}$ 是两种细菌混合液的 $\mathrm{OD}_{600}$ 值.

\section{2 实验结果}

\section{1 附生细菌的分子鉴定}

从 4 种群体微囊藻( NJ-24、NJ-54、NJ-159 和 NJ-177) 中分离得到 18 株附生细菌, 其中 $\alpha$-变形菌纲 ( Alpha proteobacterium) 7 株, $\beta$-变形菌纲 (Beta proteobacterium) 1 株, $\gamma$-变形菌纲 (Gamma proteobacterium) 2 株,放线 菌纲 (Actinobacteria) 4 株, 纤维粘网菌纲 (Cytophagia) 2 株, 黄杆菌纲 (Flavobacteriia) 2 株. 从惠氏微囊藻、坚 实微囊藻、水华微囊藻和铜绿微囊藻分离得到的附生细菌种类有差异, 分别得到了 4、6、7 和 8 株细菌, 其中 $\alpha$-变形菌在 4 种微囊藻中都存在, 附生 $\beta$-变形菌只存在于铜绿微囊藻. 惠氏微囊藻和坚实微囊藻中未发现附 生放线菌,水华微囊藻中未发现附生纤维粘网菌,而铜绿微囊藻中未发现附生黄杆菌 (表 1).

\section{2 附生细菌的生化特性}

从 18 种附生细菌利用碳源的种类数 (图 1) 来看, 仅有 3 株细菌 ( $\alpha$-变形菌纲中的 HJX5 和 HJX9, 以及放 线菌纲中的 HJX8) 只能利用 5 种碳源, 利用碳源的范围较窄, 其他细菌利用碳源的范围较广, 都能够利用 10 种以上的碳源, 有 11 株细菌能利用 20 种以上的碳源, 包括 5 株 $\alpha$-变形菌 (HJX3、HJX11、HJX18、HJX16 和 HJX15) 、1 株 $\beta$-变形菌 HJX14、2 株放线菌 (HJX17 和 HJX19)、1 株纤维粘网菌 HJX10 以及 2 株黄杆菌 (HJX1 和 HJX21). 从表 1 和图 1 可以发现, 在能够利用 20 种以上的碳源的附生细菌中, 惠氏微囊藻中有两 株细菌 ( HJX3 和 HJX1), 坚实微囊藻中有 4 株 (HJX1、HJX3、HJX10 和 HJX11), 水华微囊藻中有 2 株 ( HJX18 和 HJX21), 铜绿微囊藻中有 5 株 (HJX16、HJX15、HJX14、HJX19 和 HJX17), 每种微囊藻群体中都含 有 2 株以上代谢潜力很高 (能利用 20 种以上碳源) 的细菌. 每种微囊藻中的大多数附生细菌都能利用 10 种 以上的碳源,也就是说, 每种微囊藻中的大多数附生细菌种类都能利用较多的有机碳源.

从能够利用每种碳源的细菌种类数 (图 2) 来看, 六大类碳源中的碳水化合物、氨基酸、羧酸、多聚物、酚 酸类和胺类均可以被附生细菌所利用. 31 种碳源中有 21 种碳源都能被 10 种以上细菌利用,其中利用丙酮酸 甲酯的细菌数量最多, 达到了 17 种, 有 5 种碳源 (D-半乳糖醛酸、D-半乳糖- $\gamma$-内酯、肝糖、4-羊基苯甲酸、腐 胺) 能够被 $5 \sim 9$ 种细菌利用,有 5 种碳源 ( D, L- $\alpha$-磷酸甘油、衣康酸、 $\gamma$-差基丁酸、2-羟基苯甲酸、苯乙基胺) 只能被 1 3 种细菌利用, 其中 2-羟基苯甲酸只能被一种细菌利用.

\section{3 附生细菌的趋化性和疏水性}

18 种附生细菌中有 12 种具有趋化性 (表 2), 六大类群的附生细菌中均存在具趋化性的种类. 在具趋化 性的细菌中, $\gamma$-变形菌纲的细菌 HJX22 趋化性最强, 其趋化环直径达到 $43.1 \mathrm{~mm}, \alpha$-变形菌纲细菌 HJX16 和 HJX18 以及黄杆菌纲细菌 HJX1 的较强, 其趋化环直径超过了 $20 \mathrm{~mm}$, 而 $\beta$-变形菌纲细菌 HJX14 的最弱, 其 趋化环直径只有 $4.7 \mathrm{~mm}$. 不具有趋化性的细菌包括 3 株 $\alpha$-变形菌 (HJX5、HJX9 和 HJX15)、2 株放线菌 ( HJX8 和 HJX20) 以及 1 株黄杆菌 (HJX21). 趋化直径超过 $20 \mathrm{~mm}$ 的附生细菌在惠氏微囊藻和坚实微囊藻中 都只有 1 株细菌(HJX1), 水华微囊藻中有 2 株(HJX18 和 HJX22), 铜绿微囊藻中有 1 株( HJX16) (表 1、2). 水华微囊藻的 7 株附生细菌中有 4 株不具有趋化性, 惠氏微囊藻附生细菌都具有趋化性, 坚实微囊藻和铜绿 微囊藻附生细菌中大多数种类都具有趋化性 (表 3 ).

18 种附生细菌中有 12 种显示疏水性 (表 2), $\alpha$-变形菌纲的细菌 HJX15 和放线菌纲的细菌 HJX17 疏水 性最强, 分别达到 79.0\% 和 78.4\%, $\alpha$-变形菌纲的细菌 HJX18 和放线菌纲的细菌 HJX19 疏水性也较强, 分别 


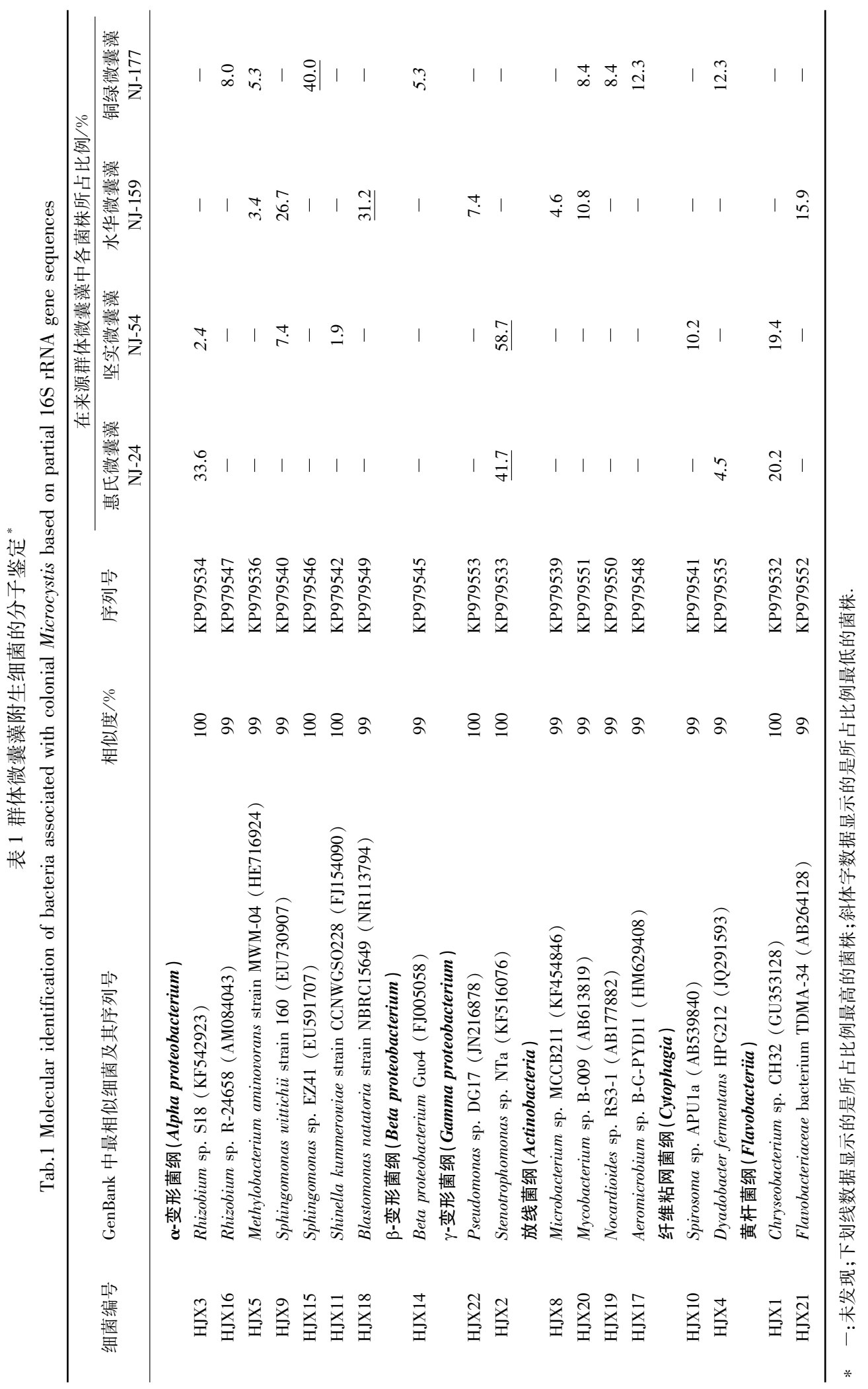




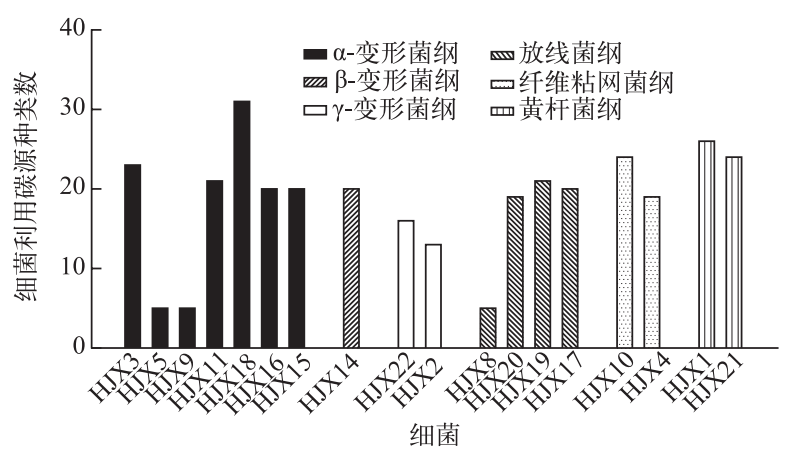

图 1 每个细菌在 BIOLOG 板上利用碳源的数量

Fig.1 The number of carbon sources utilized by each bacterial isolate in the BIOLOG system

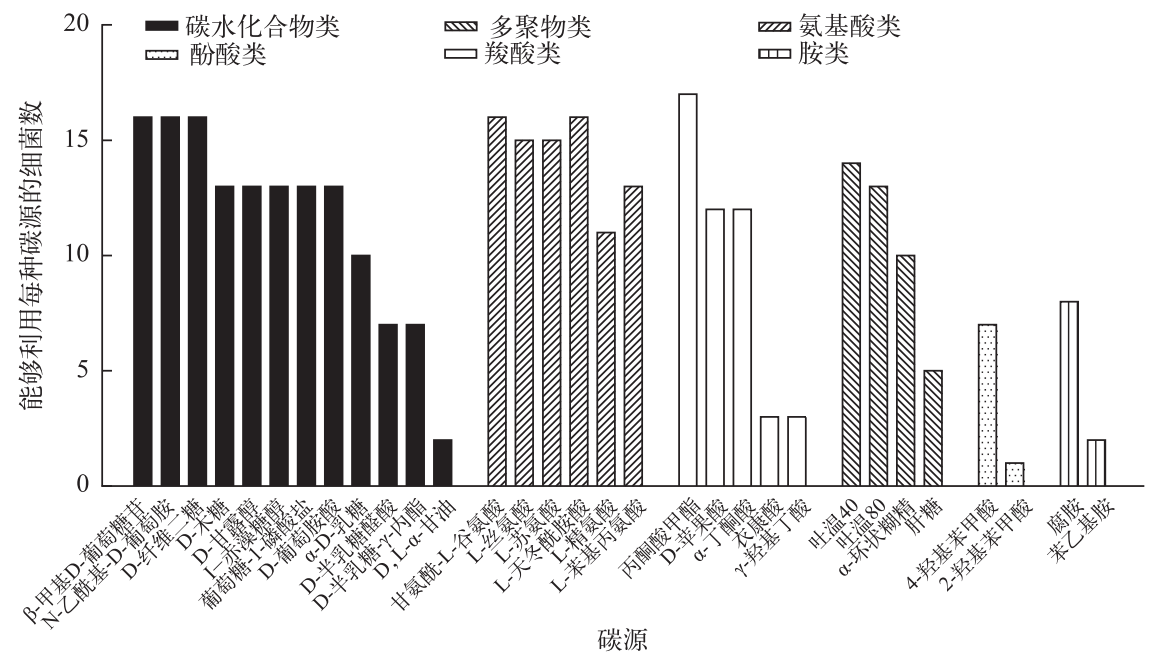

图 2 能够利用 BIOLOG 板上每种碳源的细菌数

Fig.2 The number of bacterial isolates capable of utilizing each carbon source in the BIOLOG system

为 $52.0 \%$ 和 $58.5 \% .6$ 种附生细菌疏水性数值都为 0 , 表现出亲水性,包括 $\alpha$-变形菌纲的细菌 HJX11 和 HJX16、 $\gamma-$ 变形菌纲的细菌 HJX22、纤维粘网菌纲的细菌 HJX10 以及黄杆菌纲的细菌 HJX1 和 HJX21. 在惠氏微囊藻和坚 实微囊藻中都没有疏水性大于 $50 \%$ 的附生细菌, 而在水华微囊藻中有 1 株 (HJX18), 在铜绿微囊藻中有 3 株 (HJX15、HJX19 和 HJX17) (表 1、2).每种微囊藻附生细菌中的大多数种类都具有疏水性 (表 3$)$.

\section{4 附生细菌的聚集特性}

在自聚实验过程中肉眼观察到 14 种附生细菌悬浮液中具有细菌聚集后下沉的现象, 显示具有自聚能 力 (表 2). $\alpha$-变形菌纲中的细菌 HJX5 和 HJX9 自聚能力最强, 静置 $1 \mathrm{~h}$ 后就分别达到了 $50.8 \%$ 和 $48.0 \%$, 在 静置 $4 \mathrm{~h}$ 和 $24 \mathrm{~h}$ 的自聚能力也都高于其他细菌. $\alpha$-变形菌纲的细菌 HJX16、放线菌纲的细菌 HJX8 和纤维粘 网菌纲的细菌 HJX10 自聚能力也较强,静置 $24 \mathrm{~h}$ 后都达到了 $45 \%$ 以上.在实验过程中, $\alpha$-变形菌纲的细菌 HJX3、r-变形菌纲的细菌 HJX22、纤维粘网菌纲的细菌 HJX4 和黄杆菌纲的细菌 HJX1 没有观察到细菌聚集 现象, 不具有自聚能力. 在惠氏微囊藻中没有 $24 \mathrm{~h}$ 自聚能力超过 $40 \%$ 的附生细菌, 在坚实微囊藻中有 1 株 (HJX10), 水华微囊藻中有 3 株 (HJX5、HJX9 和 HJX8), 铜绿微囊藻中有 1 株 (HJX16) (表 $1 、 2$ ). 惠氏微囊藻 的 4 株附生细菌只有 1 株具有自聚能力, 而坚实微囊藻、水华微囊藻和铜绿微囊藻附生细菌中的大多数种类 都具有自聚能力 (表 3 ). 
表 2 细菌的趋化性、疏水性与自聚能力*

Tab.2 Chemotaxis, hydrophobicity and autoaggregation of bacterial isolates

\begin{tabular}{|c|c|c|c|c|c|c|}
\hline \multirow{2}{*}{ 菌纲 } & \multirow{2}{*}{ 细菌编号 } & \multirow{2}{*}{ 趋化环直径/mm } & \multirow{2}{*}{ 疏水性/\% } & \multicolumn{3}{|c|}{ 自聚能力/\% } \\
\hline & & & & $1 \mathrm{~h}$ & $4 \mathrm{~h}$ & $24 \mathrm{~h}$ \\
\hline \multirow[t]{7}{*}{$\alpha$-变形菌纲 } & HJX3 & $13.8 \pm 0.7$ & $10.3 \pm 1.9$ & - & - & - \\
\hline & HJX5 & $0.0 \pm 0.0$ & $23.4 \pm 3.9$ & $50.8 \pm 2.3$ & $81.9 \pm 2.4$ & $86.6 \pm 3.9$ \\
\hline & HJX9 & $0.0 \pm 0.0$ & $22.1 \pm 2.1$ & $48.0 \pm 1.8$ & $67.1 \pm 3.7$ & $88.5 \pm 1.4$ \\
\hline & HJX11 & $19.2 \pm 0.4$ & $0.0 \pm 0.0$ & $1.3 \pm 0.5$ & $3.3 \pm 0.5$ & $17.4 \pm 6.0$ \\
\hline & HJX18 & $23.5 \pm 1.0$ & $52.0 \pm 0.5$ & $2.0 \pm 1.0$ & $4.5 \pm 0.7$ & $20.6 \pm 0.9$ \\
\hline & HJX16 & $28.6 \pm 0.6$ & $0.0 \pm 0.0$ & $0.8 \pm 0.3$ & $12.0 \pm 0.7$ & $48.5 \pm 1.4$ \\
\hline & HJX15 & $0.0 \pm 0.0$ & $79.0 \pm 1.8$ & $1.6 \pm 0.1$ & $4.7 \pm 0.4$ & $13.8 \pm 1.2$ \\
\hline$\beta$-变形菌纲 & HJX14 & $4.7 \pm 1.0$ & $27.9 \pm 1.9$ & $2.1 \pm 0.5$ & $11.9 \pm 4.4$ & $29.3 \pm 5.0$ \\
\hline \multirow[t]{2}{*}{$\gamma$-变形菌纲 } & HJX22 & $43.1 \pm 1.5$ & $0.0 \pm 0.0$ & - & - & - \\
\hline & HJX2 & $16.7 \pm 2.4$ & $30.7 \pm 1.7$ & $1.2 \pm 0.3$ & $4.5 \pm 0.7$ & $22.7 \pm 1.9$ \\
\hline \multirow[t]{4}{*}{ 放线菌纲 } & HJX8 & $0.0 \pm 0.0$ & $39.1 \pm 2.3$ & $9.7 \pm 1.8$ & $31.5 \pm 2.5$ & $58.5 \pm 2.2$ \\
\hline & HJX20 & $0.0 \pm 0.0$ & $19.6 \pm 1.1$ & $3.2 \pm 0.1$ & $11.0 \pm 0.3$ & $28.2 \pm 1.8$ \\
\hline & HJX19 & $14.3 \pm 0.4$ & $58.5 \pm 1.6$ & $0.8 \pm 0.2$ & $2.7 \pm 0.8$ & $16.6 \pm 1.4$ \\
\hline & HJX17 & $9.6 \pm 1.7$ & $78.4 \pm 2.9$ & $1.3 \pm 1.0$ & $3.7 \pm 1.0$ & $14.8 \pm 1.8$ \\
\hline \multirow[t]{2}{*}{ 纤维粘网菌纲 } & HJX10 & $17.3 \pm 0.5$ & $0.0 \pm 0.0$ & $5.7 \pm 1.1$ & $13.2 \pm 1.3$ & $46.4 \pm 3.1$ \\
\hline & HJX4 & $12.6 \pm 1.9$ & $27.3 \pm 4.6$ & - & - & - \\
\hline \multirow[t]{2}{*}{ 黄杆菌纲 } & HJX1 & $23.1 \pm 2.5$ & $0.0 \pm 0.0$ & - & - & - \\
\hline & HJX21 & $0.0 \pm 0.0$ & $0.0 \pm 0.0$ & $2.4 \pm 0.8$ & $4.6 \pm 0.7$ & $18.6 \pm 0.5$ \\
\hline
\end{tabular}

* 数据为平均值土标准误, $n=3$; 一表示在 $24 \mathrm{~h}$ 的实验过程中没有观察到比色血中有细菌聚集的现象.

表 34 种微囊藻中附生细菌特性的比较分析

Tab.3 Comparative analysis of characterizations of bacteria associated with four species of Microcystis

\begin{tabular}{|c|c|c|c|c|c|}
\hline 微囊藻种类 & $\begin{array}{l}\text { 分离的附生 } \\
\text { 细菌数量 }\end{array}$ & $\begin{array}{c}\text { 利用 } 10 \text { 种以上碳源 } \\
\text { 的附生细菌数量 }\end{array}$ & $\begin{array}{c}\text { 不具有趋化性的 } \\
\text { 附生细菌数量 }\end{array}$ & $\begin{array}{l}\text { 疏水值为 } 0 \text { 的 } \\
\text { 附生细菌数量 }\end{array}$ & $\begin{array}{c}\text { 不具有自聚能力的 } \\
\text { 附生细菌数量 }\end{array}$ \\
\hline 惠氏微囊藻 NJ-24 & 4 & 4 & 0 & $1($ HJX1) & 3( HJX3、HJX4 $\mathrm{HJX} 1)$ \\
\hline 坚实微囊藻 NJ-54 & 6 & 6 & 1( HJX9) & 2( HJX11 HJX10) & 1( HJX3) \\
\hline 水华微囊藻 NJ-159 & 7 & 5 & $\begin{array}{l}\text { 4( HJX5、HJX8 、 } \\
\text { HJX20、HJX21) }\end{array}$ & 2( HJX22、HJX21) & 1( HJX22) \\
\hline 铜绿微囊藻 NJ-177 & 8 & 6 & 2( HJX15、HJX20) & 1( HJX16) & 1 ( HJX4) \\
\hline
\end{tabular}

共聚分析实验发现共计有 9 组细菌表现出共聚能力 (表 4), 其他组合未发现有共聚能力, 每种微囊藻都 至少包含一组细菌具有自聚能力.来自水华微囊藻的两组细菌 HJX18 和 HJX5 以及 HJX9 和 HJX5 显示了最 高的共聚能力, 静置 $4 \mathrm{~h}$ 后分别达到了 $42.3 \%$ 和 $39.4 \%$.

\section{3 讨论}

\section{1 附生细菌的组成和生化特性}

自然界中附生细菌附着于微囊藻群体生长, 其营养来源除了藻细胞产生的有机物以外, 还可能包含水 中的其他有机物.本研究从实验室培养的微囊藻群体中分离得到的附生细菌生长完全依赖于藻细胞产生的 有机物,避免了自然环境中其他来源有机物的干扰.

分离得到的 18 株细菌中有 10 株属于变形菌门, 而且 4 种微囊藻的附生细菌群中都包含变形菌, 这与已 经报道的野外生长的微囊藻群体附生细菌群中变形菌占优势是一致的 ${ }^{[4-6]}$. 除了变形菌以外, 本研究中还分 离得到了放线菌纲、纤维粘网菌纲和黄杆菌纲的细菌, 以前的报道也显示微囊藻群体附生细菌群中包含这 些细菌 ${ }^{[4-7]}$.纤维粘网菌纲和黄杆菌纲细菌被认为可能参与了微囊藻产生的高分子化合物的降解 ${ }^{[7]}$. 附生细 
表 4 附生细菌的共聚能力*

Tab.4 Coaggregation of bacteria associated with colonial Microcystis

\begin{tabular}{lcccc}
\hline \multirow{2}{*}{ 细菌组合 } & 来源微囊藻 & \multicolumn{3}{c}{ 共聚能力 $/ \%$} \\
\cline { 3 - 4 } HJX1 和 HJX4 & 惠氏微囊藻 & $6.8 \pm 0.5$ & $4 \mathrm{~h}$ & $24 \mathrm{~h}$ \\
HJX1 和 HJX9 & 坚实微囊藻 & $4.0 \pm 0.5$ & $16.3 \pm 0.8$ & $20.6 \pm 2.5$ \\
HJX1 和 HJX10 & 坚实微囊藻 & $2.5 \pm 0.4$ & $4.2 \pm 0.7$ & $11.3 \pm 3.4$ \\
HJX18 和 HJX5 & 水华微囊藻 & $9.7 \pm 0.4$ & $13.2 \pm 0.4$ & $23.6 \pm 4.1$ \\
HJX9 和 HJX5 & 水华微囊藻 & $26.8 \pm 0.3$ & $42.3 \pm 0.3$ & $38.5 \pm 4.3$ \\
HJX16 和 HJX4 & 铜绿微囊藻 & $0.6 \pm 0.4$ & $39.4 \pm 3.6$ & $41.1 \pm 3.3$ \\
HJX19 和 HJX17 & 铜绿微囊藻 & $0.0 \pm 0.0$ & $14.5 \pm 0.4$ & $20.0 \pm 0.7$ \\
HJX20 和 HJX5 & 铜绿微囊藻 & $0.0 \pm 0.0$ & $0.1 \pm 0.1$ & $21.0 \pm 0.8$ \\
HJX5 和 HJX14 & 铜绿微囊藻 & $1.4 \pm 0.2$ & $0.0 \pm 0.0$ & $19.8 \pm 0.3$ \\
\hline
\end{tabular}

* 数据为平均值 \pm 标准误, $n=3$.

菌 HJX9 是鞘氨醇单胞菌 (Sphingomonas sp.), Park 等报道鞘氨醇单胞菌能够降解微囊藻毒素 ${ }^{[17]}$. 附生细菌 HJX22 是假单胞菌 (Pseudomonas sp.), 有报道显示假单胞菌与微囊藻细胞之间有磷的转移, 被认为是微囊藻 生长的磷库 ${ }^{[18]}$.

BIOLOG 板分析显示, 18 种附生细菌中有 15 种细菌利用碳源的范围较广, 能够利用 10 种以上碳源, BIOLOG 板中的碳水化合物、氨基酸、羧酸、多聚物、酚酸类、胺类六大类碳源都能被附生细菌所利用, 31 种碳 源中有 21 种碳源都能被 10 种及以上细菌利用. 这说明群体微囊藻的附生细菌群代谢旺盛, 具有利用藻细 胞产生的各类化合物的潜力.

\section{2 附生细菌的趋化性、细胞疏水性和聚集特性及其对细菌附着能力的影响}

铜绿假单胞菌 (Pseudomonas aeruginosa) 具有对氨基酸的趋化性, 这有利于其与鱼腥藻 (Anabaena sp.) 建 立共生关系 ${ }^{[19]}$. 研究发现 18 种附生细菌中有 12 种具有趋化性, 这一特性有利于附生细菌在微囊藻群体上 的附着生长.Prasad 等研究发现, 北极蓝藻附生细菌群中也包含一些不具有趋化性的种类 ${ }^{[14]}, 18$ 种附生细菌 中也包含 6 种不具有趋化性的细菌, 这说明虽然趋化性有利于细菌与蓝藻建立附生关系, 但是蓝藻附生细 菌群中也会包含不具有趋化性的细菌.

细胞表面疏水性是反映细菌粘附能力的一个指标 ${ }^{[2]}$, 自聚是指遗传特性相同的同种细菌之间的相互粘 附, 而共聚是指遗传特性不同的不同种细菌之间的粘附 ${ }^{[20]}$. 细菌的疏水性、自聚能力和共聚能力都有利于细 菌聚集形成群体以及粘附于生物和非生物界面 ${ }^{[20-21]}$. 微囊藻群体是由藻细胞和更多的细菌聚集形成的, 细 菌数量与藻细胞的数量比率为 $10 \sim 50^{[3]}$. 细胞疏水性已经被证明在微囊藻细胞粘附而形成群体过程中发挥 了重要作用 ${ }^{[2]}$. 分离得到的 18 种细菌中有 12 种细菌显示疏水性, 14 种细菌显示自聚能力, 每种微囊藻中也 至少有一组细菌表现出共聚能力, 这显示附生细菌的疏水性、自聚能力和共聚能力在细菌参与微囊藻群体 形成过程中可能发挥了作用.

Shi 等的研究发现微囊藻附生细菌群组成不同于水中的游离菌群 ${ }^{[4]}$, 这说明细菌会有选择性地附生于 微囊藻群体. 细菌的旺盛代谢潜力、趋化性、疏水性、自聚能力和共聚能力可能有利于附生细菌定殖于微囊藻 群体.3 株细菌 (HJX5、HJX9 和 HJX8) 利用有机物种类最少, 也不具有趋化性, 这两个方面不利于其附着生 长, 但是这 3 株细菌都具有最高的自聚能力, 高自聚能力有利于附着生长. 此外, 6 株细菌 ( HJX5、HJX9、 HJX15、HJX8、HJX20 和 HJX21) 不具有趋化性, 6 株细菌 (HJX11、HJX16、HJX22、HJX10、HJX1 和 HJX21) 都 是亲水性的, 而 4 株细菌( HJX3、HJX22、HJX4 和 HJX1) 都不具有自聚能力, 这些方面都不利于细菌附着生 长. 但是, 在趋化性、疏水性和自聚能力方面所有这些细菌都至少有一个方面的数值是不为 0 的, 也就是说, 这些细菌至少拥有一个有利于附着生长的特性.

4 株细菌 (HJX3、HJX5、HJX14 和 HJX4) 是所占比例最低的细菌 (表 1), 细菌 HJX3 和 HJX4 的自聚能力 为 0 , 细菌 HJX5 不具趋化性而且只能利用很少种类的有机碳源, 细菌 HJX14 的趋化性能很低, 这些方面应 
该是导致这 4 株细菌比例低的部分因素.3 株细菌(HJX18、HJX15 和 HJX2) 是所占比例最高的细菌(表 1),3 株细菌都能利用较多种类的有机碳源, 细菌 HJX15 具有最高的疏水性,细菌 HJX18 和 HJX2 的趋化性、疏水 性和自聚能力都不为 0 ,这些方面应该是导致这 3 株细菌比例高的部分因素.

\section{4 参考文献}

[ 1 ] Kong Fanxiang, Ma Ronghua, Gao Junfeng et al. The theory and practice of prevention, forecast and warning on cyanobacteria bloom in Lake Taihu. J Lake Sci, 2009, 21(3) : 314-328. DOI: 10.18307/2009.0302. [孔繁翔, 马荣华, 高俊峰 等. 太湖蓝藻水华的预防、预测和预警的理论与实践. 湖泊科学, 2009, 21(3): 314-328.]

[ 2 ] Yang HL, Cai YF, Xia M et al. Role of cell hydrophobicity on colony formation in Microcystis (Cyanobacteria). International Review of Hydrobiology, 2011, 96(2) : 141-148.

[ 3 ] Brunberg AK. Contribution of bacteria in the mucilage of Microcystis spp. (Cyanobacteria) to benthic and pelagic bacterial production in a hypereutrophic lake. FEMS Microbiology Ecology, 1999, 29(1) : 13-22.

[ 4 ] Shi LM, Cai YF, Kong FX et al. Specific association between bacteria and buoyant Microcystis colonies compared with other bulk bacterial communities in the eutrophic Lake Taihu, China. Environmental Microbiology Reports, 2012, 4(6) : 669-678.

[ 5 ] Shi LM, Cai YF, Wang XY et al. Community structure of bacteria associated with microcystis colonies from cyanobacterial blooms. Journal of Freshwater Ecology, 2010, 25(2) : 193-203.

[ 6 ] Shi LM, Cai YF, Li PF et al. Molecular identification of the colony-associated cultivable bacteria of the cyanobacterium Microcystis aeruginosa and their effects on algal growth. Journal of Freshwater Ecology, 2009, 24(2) : 211-218.

[ 7 ] Maruyama T, Kato K, Yokoyama A et al. Dynamics of microcystin-degrading bacteria in mucilage of Microcystis. Microbial Ecology, 2003, 46(2) : 279-288.

[ 8 ] Shen H, Niu Y, Xie P et al. Morphological and physiological changes in Microcystis aeruginosa as a result of interactions with heterotrophic bacteria. Freshwater Biology, 2011, 56(6) : 1065-1080.

[ 9 ] Rippka R. Isolation and purification of cyanobacteria. Methods in Enzymology, 1988, 167: 3-27.

[10] Reasoner DJ, Geldreich EE. A new medium for the enumeration and subculture of bacteria from potable water. Applied and Environmental Microbiology, 1985, 49(1): 1-7.

[11] Tillett D, Neilan BA. Xanthogenate nucleic acid isolation from cultured and environmental cyanobacteria. Journal of Phycology, 2000, 36(1) : 251-258.

[12] More TT, Yan S, John RP et al. Biochemical diversity of the bacterial strains and their biopolymer producing capabilities in wastewater sludge. Bioresource Technology, 2012, 121: 304-311.

[13] Mesibov R, Adler J. Chemotaxis toward amino acids in Escherichia coli. Journal of Bacteriology, 1972, 112(1) : 315.

[14] Prasad S, Pratibha MS, Manasa P et al. Diversity of chemotactic heterotrophic bacteria associated with arctic cyanobacteria. Current Microbiology, 2013, 66(1) : 64-71.

[15] Rosenberg M. Bacterial adherence to polystyrene-A replica method of screening for bacterial hydrophobicity. Applied and Environmental Microbiology, 1981, 42(2): 375-377.

[16] Kos B, Suskovic J, Vukovic S et al. Adhesion and aggregation ability of probiotic strain Lactobacillus acidophilus M92. Journal of Applied Microbiology, 2003, 94(6) : 981-987.

[17] Park HD, Sasaki Y, Maruyama T et al. Degradation of the cyanobacterial hepatotoxin microcystin by a new bacterium isolated from a hypertrophic lake. Environmental Toxicology, 2001, 16(4) : 337-343.

[18] Jiang LJ, Yang LY, Xiao L et al. Quantitative studies on phosphorus transference occuring between Microcystis aeruginosa and its attached bacterium (Pseudomonas sp.). Hydrobiologia, 2007, 581(1) : 161-165.

[19] Gallucci KK, Paerl HW. Pseudomonas aeruginosa chemotaxis associated with blooms of $\mathrm{N}_{2}$-fixing blue-green-algae (cyanobacteria). Applied and Environmental Microbiology, 1983, 45(2) : 557-562.

[20] Rickard AH, Gilbert P, High NJ et al. Bacterial coaggregation: An integral process in the development of multi-species biofilms. Trends in Microbiology, 2003, 11(2): 94-100.

[21] Garcia-Cayuela T, Korany AM, Bustos I et al. Adhesion abilities of dairy Lactobacillus plantarum strains showing an aggregation phenotype. Food Research International, 2014, 57(1) : 44-50. 